\title{
Chen Inequalities on Lightlike Hypersurface of a Lorentzian Manifold with Semi-Symmetric Metric Connection
}

\author{
Nergiz (Önen) Poyraz* , Burçin Doğan and Erol Yaşar \\ (Communicated by Yusuf Yaylı)
}

\begin{abstract}
In this paper, we introduce k-Ricci curvature and k-scalar curvature on lightlike hypersurface of a Lorentzian manifold with semi-symmetric metric connection. Using this curvatures, we establish some inequalities for lightlike hypersurface of a Lorentzian manifold with semi-symmetric metric connection. Considering these inequalities, we obtain the relation between Ricci curvature and scalar curvature endowed with semi-symmetric metric connection.
\end{abstract}

Keywords: Chen inequality; lightlike hypersurface; Lorentzian manifold; semi-symmetric metric connection.

AMS Subject Classification (2010): Primary: 53B05; 53B15; 53C40; 53C42; 53C50.

\section{Introduction}

Hayden [17] introduced a semi-symmetric metric connection on a Riemannian manifold. Imai [20] gave basic properties of a hypersurface of a Riemannian manifold with semi-symmetric metric connection and get conformal equations of Gauss and Codazzi. Konar and Biswas [22] considered semi-symmetric metric connection on Lorentz manifold. They showed that the perfect fluid space time with a non-zero constant scalar curvature which admits a semi-symmetric metric connection whose Ricci tensor is zero has vanishing expansion scalar and acceleration vector.

In 1993, Chen [9] introduced a new Riemannian invariant for a Riemannian manifold M as follows:

$$
\delta_{M}=\tau(p)-\inf (K)(p)
$$

where $\tau(p)$ is scalar curvature of $M$ and

$$
\inf (K)(p)=\left\{\inf K(\Pi): K(\Pi) \text { is a plane section of } T_{p} M\right\} .
$$

In [5], Chen established a sharp inequality for submanifold in a real space form involving intrinsic invariants, namely the sectional curvature and the scalar curvature of the submanifold; and the main extrinsic invariant, namely the squared mean curvature.

Afterwards, Chen and some geometers studied similar problems for non-degenerate submanifolds of different spaces such as in [4, 6, 8,26]. Later Mihai and Özgür in [23] studied Chen inequalities on submanifolds of real space forms endowed with semi-symmetric metric connection.

Gülbahar, Kılıç and Keleş introduced Chen-like inequalities and curvature invariants in lightlike geometry. Also, they established some inequalities between the extrinsic scalar curvatures and the intrinsic scalar curvatures [14]. In [15], they established some inequalities involving k-Ricci curvature, k-scalar curvature, the screen scalar curvature on a screen homothetic lightlike hypersurface of a Lorentzian manifold. Poyraz and Yaşar introduced k-Ricci curvature and k-scalar curvature on lightlike hypersurface of a Lorentzian product manifold with quarter-symmetric nonmetric connection and using these curvatures they established some 
Chen-type inequalities for screen homothetic lightlike hypersurface of a Lorentzian product manifold with quarter-symmetric nonmetric connection [25].

In this paper, we study inequalities for screen homothetic lightlike hypersurface of a real space form $\tilde{M}(c)$ of constant sectional curvature $c$, endowed with semi symmetric metric connection. Considering these inequalities, we obtain the relation between Ricci curvature and scalar curvature endowed with the semi symmetric metric connection.

\section{Preliminaries}

Let $M$ be a hypersurface of a $(n+1)$-dimensional, $n>1$, semi-Riemannian manifold $\widetilde{M}$ with semiRiemannian metric $\widetilde{g}$ of index $1 \leq \nu \leq n$. We consider

$$
T_{x} M^{\perp}=\left\{Y_{x} \in T_{x} \widetilde{M} \mid \widetilde{g}_{x}\left(Y_{x}, X_{x}\right)=0, \forall X_{x} \in T_{x} M\right\}
$$

for any $x \in M$. Then we say that $M$ is a lightlike (null, degenerate) hypersurface of $\widetilde{M}$ or equivalently, the immersion

$$
i: M \rightarrow \widetilde{M}
$$

of $M$ in $\widetilde{M}$ is lightlike (null, degenerate) if $T_{x} M \cap T_{x} M^{\perp} \neq\{0\}$ at any $x \in M$.

An orthogonal complementary vector bundle of $T M^{\perp}$ in $T M$ is non-degenerate subbundle of $T M$ named the screen distribution on $M$ and denoted $S(T M)$. We have the following splitting into orthogonal direct sum:

$$
T M=S(T M) \perp T M^{\perp} .
$$

The subbundle $S(T M)$ is non-degenerate, so is $S(T M)^{\perp}$, and the following satisfies:

$$
T \widetilde{M}=S(T M) \perp S(T M)^{\perp},
$$

where $S(T M)^{\perp}$ is the orthogonal complementary vector bundle to $S(T M)$ in $\left.T \widetilde{M}\right|_{M}$.

Let $\operatorname{tr}(T M)$ denote the complementary vector bundle of $T M^{\perp}$ in $S(T M)^{\perp}$. Then we have

$$
S(T M)^{\perp}=T M^{\perp} \oplus \operatorname{tr}(T M) .
$$

Let $\mathcal{U}$ be a coordinate neighborhood in $M$ and $\xi$ be a basis of $\Gamma\left(\left.T M^{\perp}\right|_{\mathcal{U}}\right)$. Then there exists a basis $N$ of $\left.\operatorname{tr}(T M)\right|_{U}$ satisfying the following conditions:

$$
\tilde{g}(N, \xi)=1,
$$

and

$$
\tilde{g}(N, N)=\tilde{g}(W, N)=0, \quad \forall W \in \Gamma\left(\left.S(T M)\right|_{\mathcal{U}}\right) .
$$

The subbundle $\operatorname{tr}(T M)$ is named a lightlike transversal vector bundle of $M$. We note that $\operatorname{tr}(T M)$ is never orthogonal to $T M$. From (2.1), (2.2) and (2.3) we have

$$
\left.T \widetilde{M}\right|_{M}=S(T M) \perp\left(T M^{\perp} \oplus \operatorname{tr}(T M)\right)=T M \oplus \operatorname{tr}(T M),
$$

$[11,16]$

\section{Semi-Symmetric Metric Connection}

For $n>1$, let $\widetilde{M}$ be an $(n+2)$-dimensional differentiable manifold of class $C^{\infty}$ and $\widetilde{\nabla}$ a linear connection in $\widetilde{M}$. The torsion tensor $\widetilde{T}$ of $\widetilde{\nabla}$ is given by

$$
\widetilde{T}(\widetilde{X}, \widetilde{Y})=\widetilde{\nabla}_{\widetilde{X}} \widetilde{Y}-\widetilde{\nabla}_{\widetilde{Y}} \widetilde{X}-[\widetilde{X}, \widetilde{Y}], \forall \widetilde{X}, \widetilde{Y} \in \Gamma(T \widetilde{M})
$$


and have type $(1,2)$. When the torsion tensor $\widetilde{T}$ satisfies

$$
\widetilde{T}(\tilde{X}, \tilde{Y})=\widetilde{\pi}(\widetilde{Y}) \widetilde{X}-\tilde{\pi}(\tilde{X}) \widetilde{Y}
$$

for a 1 -form $\widetilde{\pi}$, the connection $\widetilde{\nabla}$ is said to be semi-symmetric (see [27]).

Let us consider a semi-Riemannian metric $\widetilde{g}$ of index $\nu$ with $1 \leq \nu \leq n+1$ in $\widetilde{M}$ and $\widetilde{\nabla}$ satisfying

$$
\widetilde{\nabla} \widetilde{g}=0 .
$$

A linear connection of this type is called a metric connection (see [23]).

We assume that the semi-Riemannian manifold $\widetilde{M}$ admits a semi-symmetric metric connection which is given by

$$
\widetilde{\nabla}_{\widetilde{X}} \widetilde{Y}=\stackrel{\check{\nabla}}{\widetilde{X}}_{\widetilde{Y}}+\widetilde{\pi}(\widetilde{Y}) \widetilde{X}-\widetilde{g}(\widetilde{X}, \widetilde{Y}) \tilde{Q}
$$

for arbitrary vector fields $\widetilde{X}$ and $\widetilde{Y}$ of $\widetilde{M}$, where $\stackrel{\circ}{\nabla}$ denotes the Levi-Civita connection with respect to the semi-Riemannian metric $\widetilde{g}, \tilde{\pi}$ is a $1-$ form and $\tilde{Q}$ is the vector field defined by

$$
\widetilde{g}(\tilde{Q}, \widetilde{X})=\widetilde{\pi}(\widetilde{X})
$$

for an arbitrary vector field $\widetilde{X}$ of $\widetilde{M}$ (see [13] and [27]).

The Gauss formula with respect to the induced connection $\nabla$ on the lightlike hypersurface from the semisymmetric metric connection $\widetilde{\nabla}$ is given by

$$
\widetilde{\nabla}_{X} Y=\nabla_{X} Y+m(X, Y) N
$$

for arbitrary vector fields $X$ and $Y$ of $M$, where $m$ is a tensor of type $(0,2)$ of the lightlike hypersurface of $M$ [28].

On the other hand, denoting the projection of $T M$ on $S(T M)$ with respect to the decomposition (2.1) by $P$, one has the Weingarten formula with respect to the semi-symmetric connection which is given by

$$
\nabla_{X} P Y=\stackrel{*}{\nabla}_{X} P Y+D(X, P Y) \xi
$$

where $\stackrel{*}{\nabla}_{X} P Y$ belongs to $\Gamma(S(T M))$ and $D$ is $1-$ form on $M$.

The curvature tensor $\stackrel{\circ}{\widetilde{R}}$ with respect to $\stackrel{\circ}{\widetilde{\nabla}}$ on real space form $\widetilde{M}(c)$ is defined by

$$
\stackrel{\circ}{\widetilde{R}}(X, Y, Z, W)=c\{g(X, W) g(Y, Z)-g(Y, W) g(X, Z)\} .
$$

Using (3.1), for any vector fields $X, Y, Z, W \in \Gamma(T M)$ and $(0,2)$ tensor field $\alpha$ which defined by

$$
\alpha(X, Y)=\left(\stackrel{\circ}{\nabla}_{X} \pi\right) Y-\pi(X) \pi(Y)+\frac{1}{2} \pi(Q) g(X, Y)
$$

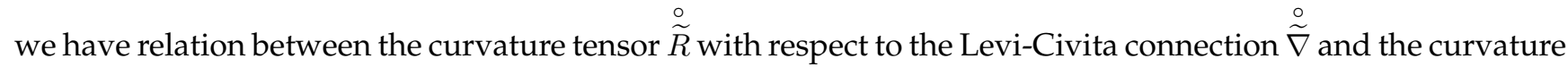
tensor $\widetilde{R}$ with respect to the semi-symmetric metric connection $\widetilde{\nabla}$ given by

$$
\begin{aligned}
\widetilde{R}(X, Y, Z, W)= & \stackrel{\circ}{\widetilde{R}}(X, Y, Z, W)-\alpha(Y, Z) g(X, W)+\alpha(X, Z) g(Y, W) \\
& -\alpha(X, W) g(Y, Z)+\alpha(Y, W) g(X, Z),
\end{aligned}
$$

[19].

Moreover, Gauss-Codazzi equations with respect to the semi-symmetric metric connection $\widetilde{\nabla}$ on $\widetilde{M}$ can be written as [28]

$$
\begin{aligned}
R(X, Y, Z, P W)= & \widetilde{R}(X, Y, Z, P W)-m(X, Z) D(Y, P W)+m(Y, Z) D(X, P W) \\
& -\{m(X, Z) \eta(Y)-m(Y, Z) \eta(X)\} \pi(P W)
\end{aligned}
$$




$$
\begin{aligned}
\tilde{g}(\tilde{R}(X, Y) Z, \xi)= & \pi(Y) m(X, Z)-\pi(X) m(Y, Z)+\left(\nabla_{X} m\right)(Y, Z)-\left(\nabla_{Y} m\right)(X, Z) \\
& +m(Y, Z)(\tau(X)-\mu \eta(X))-m(X, Z)(\tau(Y)-\mu \eta(Y)),
\end{aligned}
$$

and

$$
\widetilde{g}(\tilde{R}(X, Y) Z, N)=g(R(X, Y) Z, N),
$$

for any vector fields $X, Y, Z, W \in \Gamma(T M)$.

From (3.4), (3.6) and (3.7), we have

$$
\begin{aligned}
R(X, Y, Z, P W)= & c\{g(Y, Z) g(X, P W)-g(X, Z) g(Y, P W)\} \\
& -\alpha(Y, Z) g(X, P W)+\alpha(X, Z) g(Y, P W) \\
& -\alpha(X, W) g(Y, Z)+\alpha(Y, W) g(X, Z) \\
& -m(X, Z) D(Y, P W)+m(Y, Z) D(X, P W) \\
& -\{m(X, Z) \eta(Y)-m(Y, Z) \eta(X)\} \pi(P W) .
\end{aligned}
$$

Denote by $\lambda$ the trace of $\alpha$.

Let $(M, g, S(T M))$ be a lightlike hypersurface of a semi-Riemannian manifold $(\widetilde{M}, \widetilde{g})$. Then M is named totally umbilical lightlike hypersurface if there exists a smooth function such that

$$
m(X, Y)_{p}=H g_{p}(X, Y), \quad X, Y \in \Gamma\left(T_{p} M\right)
$$

for any coordinate neighborhood $U$ and $X, Y \in \Gamma\left(T M_{\left.\right|_{U}}\right)$, where $H \in R$. If every points of $M$ is umbilical, the lightlike hypsersurface $M$ is named totally umbilical in $\widetilde{M}$ [11]. If $m=0$, then the lightlike hypsersurface $M$ is named totally geodesic in $\widetilde{M}$.

The mean curvature $\mu$ of $M$ with respect to an orthonormal basis $\left\{e_{1}, \ldots, e_{n}\right\}$ of $\Gamma(S(T M))$ is defined by

$$
\mu=\frac{1}{n} \operatorname{tr}(m)=\frac{1}{n} \sum_{i=1}^{n} \varepsilon_{i} m\left(e_{i}, e_{i}\right), \quad g\left(e_{i}, e_{i}\right)=\varepsilon_{i} .
$$

A lightlike hypersurface $(M, g)$ of a semi-Riemannian manifold $(\widetilde{M}, \widetilde{g})$ is called screen locally conformal if the shape operators $A_{N}$ and $\stackrel{*}{A}_{\xi}$ of $M$ and $S(T M)$, respectively, are related by

$$
A_{N}=\varphi \stackrel{*}{A},
$$

where $\varphi$ is a non-vanishing smooth function on a neighborhood $\mathcal{U}$ on $M$. In particular, , if $\varphi$ is a non-zero constant, $\mathrm{M}$ is called screen homothetic [12].

Let $M$ be a two-dimensional non-degenerate plane. The sectional curvature at $p \in M$ is given by

$$
K_{i j}=\frac{g\left(R\left(e_{j}, e_{i}\right) e_{i}, e_{j}\right)}{g\left(e_{i}, e_{i}\right) g\left(e_{j}, e_{j}\right)-g\left(e_{i}, e_{j}\right)^{2}}
$$

[12].

Let $p \in M$ and $\xi$ be null vector of $T_{p} M$. A plane $\Pi$ of $T_{p} M$ is said to be null plane if it contains $\xi$ and $e_{i}$ such that $g\left(\xi, e_{i}\right)=0$ and $g\left(e_{i}, e_{i}\right)=\varepsilon_{i}= \pm 1$. One defines the null sectional curvature of $\Pi$ by

[2].

$$
K_{i}^{\text {null }}=\frac{g\left(R_{p}\left(e_{i}, \xi\right) \xi, e_{i}\right)}{g_{p}\left(e_{i}, e_{i}\right)}
$$

We denote the Ricci tensor of $\widetilde{M}$ with $\widetilde{R i c}$ and the induced Ricci type tensor of $M$ with $R^{(0,2)}$. Then, $\widetilde{R i c}$ and $R^{(0,2)}$ are given by

$$
\begin{aligned}
\widetilde{\operatorname{Ric}}(X, Y) & =\operatorname{trace}\{Z \rightarrow \widetilde{R}(Z, X) Y\}, \quad \forall X, Y \in \Gamma(T \widetilde{M}), \\
R^{(0,2)}(X, Y) & =\operatorname{trace}\{Z \rightarrow R(Z, X) Y\}, \quad \forall X, Y \in \Gamma(T M),
\end{aligned}
$$

where

$$
R^{(0,2)}(X, Y)=\sum_{i=1}^{n} \varepsilon_{i} g\left(R\left(e_{i}, X\right) Y, e_{i}\right)+\tilde{g}(R(\xi, X) Y, N)
$$


for the quasi-orthonormal frame $\left\{e_{1}, \ldots, e_{n}, \xi\right\}$ of $T_{p} M$.

Scalar curvature $\tau$ is defined

$$
\tau(p)=\sum_{i, j=1}^{n} K_{i j}+\sum_{i=1}^{n} K_{i}^{\text {null }}+K_{i N},
$$

where $K_{i N}=\tilde{g}\left(R\left(\xi, e_{i}\right) e_{i}, N\right)$ for $i \in\{1, \ldots, n\}[10]$.

If $\operatorname{dim}(M)>2$ and

$$
\operatorname{Ric}(X, Y)=k g(X, Y),
$$
[12].

then $M$ is an Einstein manifold. For $\operatorname{dim}(M)=2$, any $M$ is Einstein but $k$ in (3.18) is not necessarily constant

\section{Chen Ricci Inequality}

In this section, we use the same notations and terminologies as in [14].

Let $M$ be an $(n+1)$-dimensional lightlike hypersurface of a Lorentzian manifold $\widetilde{M}$ with a semi-symmetric metric connection. $\left\{e_{1}, \ldots ., e_{n}, \xi\right\}$ and $\left\{e_{1}, \ldots ., e_{n}\right\}$ are basis of $\Gamma(T M)$ and an orthonormal basis of $\Gamma(S(T M))$, respectively. Similarly, for $k \leq n, \pi_{k, \xi}=s p\left\{e_{1}, \ldots, e_{k}, \xi\right\}$ and $\pi_{k}=s p\left\{e_{1}, \ldots, e_{k}\right\}$ are $(k+1)$-dimensional degenerate plane section and $\pi_{k}=s p\left\{e_{1}, \ldots ., e_{k}\right\}$ is $k$-dimensional non-degenerate plane section, respectively. For a unit vector $X \in \Gamma(T M)$, the $k$-degenerate Ricci curvature and the $k$-Ricci curvature are defined by

$$
\begin{gathered}
\operatorname{Ric}_{\pi_{k, \xi}}(X)=R^{(0,2)}(X, X)=\sum_{j=1}^{k} g\left(R\left(e_{j}, X\right) X, e_{j}\right)+\widetilde{g}(R(\xi, X) X, N), \\
\operatorname{Ric}_{\pi_{k}}(X)=R^{(0,2)}(X, X)=\sum_{j=1}^{k} g\left(R\left(e_{j}, X\right) X, e_{j}\right),
\end{gathered}
$$

respectively [14]. Also for $p \in M, k$-degenerate scalar curvature and $k$-scalar curvature are determined by

$$
\begin{gathered}
\tau_{\pi_{k, \xi}}(p)=\sum_{i, j=1}^{k} K_{i j}+\sum_{i=1}^{k} K_{i}^{\text {null }}+K_{i N}, \\
\tau_{\pi_{k}}(p)=\sum_{i, j=1}^{k} K_{i j},
\end{gathered}
$$

respectively [14]. For $k=n, \pi_{n}=s p\left\{e_{1}, \ldots ., e_{n}\right\}=\Gamma(S(T M))$, we have the screen Ricci curvature and the screen scalar curvature given by

$$
\operatorname{Ric}_{S(T M)}\left(e_{1}\right)=\operatorname{Ric}_{\pi_{n}}\left(e_{1}\right)=\sum_{j=1}^{n} K_{1 j}=K_{12}+\ldots+K_{1 n},
$$

and

$$
\tau_{S(T M)}=\sum_{i, j=1}^{n} K_{i j}
$$

respectively [14].

Using (3.10) we obtain

$$
\tau_{S(T M)}(p)=n(n-1) c-2(n-1) \lambda+\sum_{i, j=1}^{n} m_{i i} D_{j j}-m_{i j} D_{j i}
$$

where $\lambda$ is the trace of $\alpha$ and $m_{i j}=m\left(e_{i}, e_{j}\right), D_{i j}=D\left(e_{i}, e_{j}\right)$ for $i, j \in\{1, \ldots, n\}$. 
Let $\widetilde{M}(c)$ be a Lorentzian space form and $M$ be a screen homothetic lightlike hypersurface of an $(n+2)$ dimensional $\widetilde{M}(c)$. Using (3.6)-(3.10) we get the following equations:

$$
\begin{aligned}
& \tau_{S(T M)}(p)=n(n-1) c-2(n-1) \lambda+\varphi n^{2} \mu^{2}-\varphi \sum_{i, j=1}^{n}\left(m_{i j}\right)^{2} \\
& \sum_{i=1}^{n} K_{i}^{\text {null }}=\sum_{i=1}^{n} R\left(e_{i}, \xi, \xi, e_{i}\right) \\
&=\sum_{i=1}^{n} \widetilde{R}\left(e_{i}, \xi, \xi, e_{i}\right) \\
&=\sum_{i=1}^{n}-\alpha(\xi, \xi)=-n \alpha(\xi, \xi), \\
& \sum_{i=1}^{n} K_{i}^{N}=\sum_{i=1}^{n} R\left(\xi, e_{i}, e_{i}, N\right) \\
&= \sum_{i=1}^{n} \widetilde{R}\left(\xi, e_{i}, e_{i}, N\right) \\
&= \sum_{i=1}^{n}\left(c-\alpha(\xi, N)-\alpha\left(e_{i}, e_{i}\right)\right) \\
&=n c-n \alpha(\xi, N)-\lambda .
\end{aligned}
$$

From (3.17), (4.8), (4.9) and (4.10), we get the induced scalar curvature $\tau(p)$ of $M$ as following:

$$
\tau(p)=n^{2} c-2(n+1) \lambda+\varphi n^{2} \mu^{2}-\varphi \sum_{i, j=1}^{n}\left(m_{i j}\right)^{2}-n(\alpha(\xi, \xi)+\alpha(\xi, N)) .
$$

Using (4.11) we obtain the following :

Theorem 4.1. Let $M$ be an $(n+1)$-dimensional screen homothetic lightlike hypersurface with $\varphi>0$ of a Lorentzian space form $\widetilde{M}(c)$ of constant sectional curvature $c$, endowed with a semi-symmetric metric connection $\widetilde{\nabla}$. Then we have

$$
\frac{1}{\varphi}\left(\tau(p)-n^{2} c+2(n+1) \lambda+n(\alpha(\xi, \xi)+\alpha(\xi, N))\right) \leq n^{2} \mu^{2}
$$

The equality of (4.12) holds for $p \in M$ if and only if $p$ is a totally geodesic point..

Lemma 4.1. [26] Let $a_{1}, a_{2}, \ldots, a_{n}$, be n-real number $(n>1)$, then

$$
\frac{1}{n}\left(\sum_{i=1}^{n} a_{i}\right)^{2} \leq \sum_{i=1}^{n} a_{i}^{2}
$$

with equality iff $a_{1}=a_{2}=\ldots=a_{n}$.

Theorem 4.2. Let $M$ be an $(n+1)$-dimensional screen homothetic lightlike hypersurface with $\varphi>0$ of a Lorentzian space form $\widetilde{M}(c)$ of constant sectional curvature $c$, endowed with a semi-symmetric metric connection $\widetilde{\nabla}$. Then we have

$$
\frac{1}{\varphi}\left(\tau(p)-n^{2} c+2(n+1) \lambda+n(\alpha(\xi, \xi)+\alpha(\xi, N))\right) \leq n(n-1) \mu^{2} .
$$

For $p \in M$ the equality of (4.13) satisfies iff $p$ is a totally umbilical point. 
Proof. Using Lemma 4.1 one derives

$$
n \mu^{2} \leq \sum_{i=1}^{n}\left(m_{i i}\right)^{2} .
$$

After substituting (4.14) in (4.11) we find (4.13). For $p \in M$ the equality of (4.13) satisfies iff

$$
m_{11}=\ldots=m_{n n} .
$$

Thus $p$ is a totally umbilical point.

If the sectional curvature is screen homothetic, then the sectional curvature of lightlike hypersurface is symmetric. One defines the screen scalar curvature $r_{S(T M)}$

$$
r_{S(T M)}(p)=\sum_{1 \leq i<j \leq n} K_{i j}=\frac{1}{2} \sum_{i, j=1}^{n} K_{i j}=\frac{1}{2} \tau_{S(T M)}(p) .
$$

By using (4.8), the equality (4.15) can be rewritten as follows:

$$
2 r_{S(T M)}(p)=n(n-1) c-2(n-1) \lambda+\varphi n^{2} \mu^{2}-\sum_{i, j=1}^{n}\left(m_{i j}\right)^{2} .
$$

Theorem 4.3. Let $M$ be an $(n+1)$-dimensional screen homothetic lightlike hypersurface with $\varphi>0$ of a Lorentzian space form $\widetilde{M}(c)$ of constant sectional curvature $c$, endowed with a semi-symmetric metric connection $\widetilde{\nabla}$ such that the vector field $P$ is tangent to $M$. Then, the following statements are true.

(i) For $X \in S^{1}(T M)=\{X \in S(T M):\langle X, X\rangle=1\}$

$$
\operatorname{Ric}_{S(T M)}(X) \leq \frac{1}{4} \varphi n^{2} \mu^{2}+(n-1) c-(2 n-3) \lambda+(n-2) \alpha(X, X) .
$$

(ii) The equality case of (4.17) is satisfied by $X \in T_{p}^{1}(M)$ iff

$$
\begin{aligned}
& m(X, Y)=0, \text { for all } Y \in T_{p}(M) \text { orthogonal to } X, \\
& m(X, X)=\frac{n}{2} \mu .
\end{aligned}
$$

(iii) The equality case of (4.17) holds for all $X \in T_{p}^{1}(M)$ iff either $p$ is a totally geodesic point or $n=2$ and $p$ is a totally umbilical point.

Proof. From (4.16), we get

$$
\begin{aligned}
\frac{1}{4} \varphi n^{2} \mu^{2}= & r_{S(T M)}(p)-\frac{n(n-1)}{2} c+(n-1) \lambda+\frac{1}{4} \varphi\left(m_{11}-m_{22}-\ldots-m_{n n}\right)^{2} \\
& +\varphi \sum_{j=2}^{n}\left(m_{1 j}\right)^{2}-\varphi \sum_{2 \leq i<j \leq n} m_{i i} m_{j j}-\left(m_{i j}\right)^{2} .
\end{aligned}
$$

Using (3.10) we obtain

$$
\varphi \sum_{2 \leq i<j \leq n} m_{i i} m_{j j}-\left(m_{i j}\right)^{2}=\sum_{2 \leq i<j \leq n} K_{i j}-\frac{(n-2)(n-1)}{2} c+(n-2)\left(\lambda-\alpha\left(e_{1}, e_{1}\right)\right) .
$$

From (4.19) and (4.20), we have

$$
\begin{aligned}
\operatorname{Ric}_{S(T M)}\left(e_{1}\right)= & \frac{1}{4} \varphi n^{2} \mu^{2}+(n-1) c-(2 n-3) \lambda-\frac{1}{4} \varphi\left(m_{11}-m_{22}-\ldots-m_{n n}\right)^{2} \\
& -\varphi \sum_{j=2}^{n}\left(m_{1 j}\right)^{2}+(n-2) \alpha\left(e_{1}, e_{1}\right) .
\end{aligned}
$$


If we take $e_{1}=X$ like any vector of $T_{p}^{1}(M)$ in (4.21) one gets (4.17).

Equality holds in (4.17) for $X \in T_{p}^{1}(M)$ iff

$$
m_{12}=m_{13}=\ldots=m_{1 n}=0 \text { and } m_{11}=m_{22}+\ldots+m_{n n},
$$

which is quivalent to (4.18). we have

Now we prove the statement (iii). Assuming the equality in (4.17) for all $X \in T_{p}^{1}(M)$, in view of (4.22),

$$
\begin{gathered}
m_{i j}=0, \quad i \neq j . \\
2 m_{i i}=m_{11}+m_{22}+\ldots+m_{n n}, \quad i \in\{1, \ldots, n\} .
\end{gathered}
$$

From (4.24), we have $2 m_{11}=2 m_{22}=\ldots=2 m_{n n}=m_{11}+m_{22}+\ldots+m_{n n}$, which implies that

$$
(n-2)\left(m_{11}+m_{22}+\ldots+m_{n n}\right)=0 .
$$

Thus, either $m_{11}+m_{22}+\ldots+m_{n n}=0$ or $n=2$. If $m_{11}+m_{22}+\ldots+m_{n n}=0$, then from (4.24), we get

$$
m_{i i}=0 \text { for all } i \in\{1, \ldots, n\} .
$$

By the above equation and (4.23), we obtain $m_{i j}=0$ for all $i, j \in\{1, \ldots, n\}$, that imlies that $p$ is a totally geodesic point. If $n=2$, then from (4.24), $2 m_{11}=2 m_{22}=m_{11}+m_{22}$, that is, $p$ is a totally umbilical point. Converse is trivial.

Lemma 4.2. If $n>k \geq 2$ and $a_{1}, \ldots, a_{n} \in \mathbb{R}$ are real numbers such that

$$
\left(\sum_{i=1}^{n} a_{i}\right)^{2}=(n-k+1)\left(\sum_{i=1}^{n} a_{i}^{2}+a\right),
$$

then

$$
2 \sum_{1 \leq i<j \leq k}^{n} a_{i} a_{j} \geq a .
$$

with equality holding iff

$$
a_{1}+a_{2}+\ldots+a_{k}=a_{k+1}=\ldots=a_{n} .
$$

Theorem 4.4. Let $M$ be an $(n+1)$-dimensional screen homothetic lightlike hypersurface with $\varphi>0$ of a Lorentzian space form $\widetilde{M}(c)$ of constant sectional curvature $c$, endowed with a semi-symmetric metric connection $\widetilde{\nabla}$ such that the vector field $P$ is tangent to $M$. Then, for each point $p \in M$ and each non-degenerate $k$-plane section $\Pi_{k} \subset T p M$ $(n>k \geq 2)$, we have

$$
\begin{aligned}
\tau_{S(T M)}(p)-\tau\left(\pi_{k}\right) \geq & (n-k)\left(\frac{\varphi n^{2}}{(n-k+1)} \mu^{2}+(n-k+1) c-\lambda\right) \\
& -\varphi \sum_{r=k}^{n}\left(m_{i i}\right)^{2}+2(k-1) \operatorname{trace}\left(\left.\alpha\right|_{\pi_{k}}\right) .
\end{aligned}
$$

If the equality case of (4.25) satisfies for $p \in M$, thus $M$ is minimal and the form of shape operator of $M$ becomes

$$
A_{\xi}^{*}=\left[\begin{array}{cccccc}
m_{11} & m_{12} & \cdot & \cdot & m_{1 k} & \\
m_{21} & m_{22} & \cdot & \cdot & m_{2 k} & \\
\cdot & \cdot & \cdot & \cdot & \cdot & 0 \\
\cdot & \cdot & \cdot & \cdot & \cdot & \\
\cdot & \cdot & \cdot & \cdot & \cdot & \\
m_{k 1} & m_{k 2} & \cdot & \cdot & -\sum_{i=1}^{k-1}\left(m_{i i}\right) & \\
& & 0 & & & 0_{n-k}
\end{array}\right]
$$


Proof. One takes

$$
\varepsilon=\tau_{S(T M)}(p)-n(n-1) c+2(n-1) \lambda-\varphi \frac{n^{2}(n-k)}{(n-k+1)} \mu^{2},
$$

in (4.8), then we have

$$
\varepsilon=\varphi \frac{n^{2}}{(n-k+1)} \mu^{2}-\varphi \sum_{i, j=1}^{n}\left(m_{i j}\right)^{2}
$$

Therefore, we can write

$$
\left(\sum_{i=1}^{n} m_{i i}\right)^{2}=(n-k+1)\left(\sum_{i=1}^{n}\left(m_{i i}\right)^{2}+\sum_{i \neq j=1}^{n}\left(m_{i j}\right)^{2}+\frac{\varepsilon}{\varphi}\right) .
$$

From Lemma 4.2 we get

$$
2 \sum_{1 \leq i<j \leq k} m_{i i} m_{j j} \geq \sum_{i \neq j=1}^{n}\left(m_{i j}\right)^{2}+\frac{\varepsilon}{\varphi} .
$$

Now, a non-degenerate plane section $\pi_{k}$ spanned by $\left\{e_{1}, e_{2}, \ldots, e_{k}\right)$. Then one obtains

$$
\begin{aligned}
\tau\left(\pi_{k}\right)= & k(k-1) c-\sum_{i, j=1}^{k}\left(\alpha\left(e_{i}, e_{i}\right)+\alpha\left(e_{j}, e_{j}\right)\right)+\varphi \sum_{i, j=1}^{k} m_{i i} m_{j j}-\left(m_{i j}\right)^{2} \\
= & k(k-1) c-\sum_{i, j=1}^{k}\left(\alpha\left(e_{i}, e_{i}\right)+\alpha\left(e_{j}, e_{j}\right)\right)+\varphi \sum_{i=1}^{k}\left(m_{i i}\right)^{2} \\
& +2 \varphi \sum_{1 \leq i<j \leq k} m_{i i} m_{j j}-\varphi \sum_{i, j=1}^{k}\left(m_{i j}\right)^{2} \\
\geq & k(k-1) c-2(k-1) \sum_{i=1}^{k} \alpha\left(e_{i}, e_{i}\right)+\varepsilon+\sum_{i \neq j=1}^{n}\left(m_{i j}\right)^{2}-\varphi \sum_{i, j=1}^{k}\left(m_{i j}\right)^{2} \\
\geq & k(k-1) c-2(k-1) \sum_{i=1}^{k} \alpha\left(e_{i}, e_{i}\right)+\varepsilon+\varphi \sum_{i, j=1}^{n}\left(m_{i j}\right)^{2}-\varphi \sum_{i=1}^{n}\left(m_{i i}\right)^{2}-\varphi \sum_{i, j=1}^{k}\left(m_{i j}\right)^{2} \\
\geq & k(k-1) c-2(k-1) \sum_{i=1}^{k} \alpha\left(e_{i}, e_{i}\right)+\varepsilon+\varphi \sum_{i, j=k+1}^{n}\left(m_{i j}\right)^{2}-\varphi \sum_{i=k}^{n}\left(m_{i i}\right)^{2} .
\end{aligned}
$$

We remark that

$$
\sum_{i=1}^{k} \alpha\left(e_{i}, e_{i}\right)=\lambda-\operatorname{trace}\left(\left.\alpha\right|_{\pi_{k}}\right) .
$$

Using (4.27), (4.30) and (4.31) we get

$$
\begin{aligned}
\tau\left(\pi_{k}\right) \geq & k(k-1) c-2(k-1)\left(\lambda-\operatorname{trace}\left(\left.\alpha\right|_{\pi_{k}}\right)\right)-\varphi \sum_{i=k}^{n}\left(m_{i i}\right)^{2} \\
& +\tau_{S(T M)}(p)-n(n-1) c+2(n-1) \lambda-\varphi \frac{n^{2}(n-k)}{n-k+1} \mu^{2} .
\end{aligned}
$$

From (4.32) we have (4.25) and (4.26) which implies that $M$ is minimal.

Furthermore, the second fundamental form $m$ and the screen second fundamental form $D$ provide

$$
\sum_{i, j=1}^{n} m_{i j} D_{j i}=\frac{1}{2}\left\{\sum_{i, j=1}^{n}\left(m_{i j}+D_{j i}\right)^{2}-\sum_{i, j=1}^{n}\left(m_{i j}\right)^{2}+\left(D_{j i}\right)^{2}\right\}
$$


and

$$
\sum_{i, j=1}^{n} m_{i i} D_{j j}=\frac{1}{2}\left\{\left(\sum_{i, j=1}^{n} m_{i i}+D_{j j}\right)^{2}-\left(\sum_{i=1}^{n} m_{i i}\right)^{2}-\left(\sum_{j=1}^{n} D_{j j}\right)^{2}\right\} .
$$

Theorem 4.5. Let $M$ be an $(n+1)$-dimensional lightlike hypersurface of a Lorentzian space form

$\widetilde{M}(c)$ of constant sectional curvature $c$, endowed with a semi-symmetric metric connection $\widetilde{\nabla}$. Then, we have

(i)

$$
\tau_{S(T M)}(p) \leq n(n-1) c-2(n-1) \lambda+n \mu \text { trace } A_{N}+\frac{1}{2} \sum_{i, j=1}^{n}\left(m_{i j}\right)^{2}+\left(D_{j i}\right)^{2} .
$$

The equality case of (4.35) satisfies for all $p \in M$ iff either $M$ is a screen homothetic lightlike hypersurface with $\varphi=-1$ or $M$ is a totally geodesic lightlike hypersurface.

(ii)

$$
\tau_{S(T M)}(p) \geq n(n-1) c-2(n-1) \lambda+n \mu \text { trace } A_{N}-\frac{1}{2} \sum_{i, j=1}^{n}\left(m_{i j}\right)^{2}+\left(D_{j i}\right)^{2} .
$$

The equality case of (4.36) satisfies for all $p \in M$ iff either $M$ is a screen homothetic lightlike hypersurface with $\varphi=1$ or $M$ is a totally geodesic lightlike hypersurface.

(iii) (4.35) and (4.36) with equalities iff $p$ is a totally geodesic point.

Proof. From (4.7) and (4.33), we get

$$
\tau_{S(T M)}(p)=n(n-1) c-2(n-1) \lambda+\sum_{i, j=1}^{n} m_{i i} D_{j j}-\frac{1}{2} \sum_{i, j=1}^{n}\left(m_{i j}+D_{j i}\right)^{2}+\frac{1}{2} \sum_{i, j=1}^{n}\left(m_{i j}\right)^{2}+\left(D_{j i}\right)^{2}
$$

which yields (4.35).

Since

$$
\frac{1}{2}\left(\left(m_{i j}\right)^{2}+\left(D_{j i}\right)^{2}\right)=\frac{1}{4}\left(m_{i j}+D_{j i}\right)^{2}+\frac{1}{4}\left(m_{i j}-D_{j i}\right)^{2},
$$

one obtains

$$
\tau_{S(T M)}(p)=n(n-1) c-2(n-1) \lambda+\sum_{i, j=1}^{n} m_{i i} D_{j j}+\frac{1}{2} \sum_{i, j=1}^{n}\left(m_{i j}-D_{j i}\right)^{2}-\frac{1}{2} \sum_{i, j=1}^{n}\left(m_{i j}\right)^{2}+\left(D_{j i}\right)^{2}
$$

which implies (4.36). From (4.35), (4.36), (4.37) and (4.39) (i), (ii) and (iii) statements are easily obtained.

Thus we get the following corollary.

Corollary 4.1. Let $M$ be an $(n+1)$-dimensional screen homothetic lightlike hypersurface of a Lorentzian space form $\widetilde{M}(c)$ of constant sectional curvature $c$, endowed with a semi-symmetric metric connection $\widetilde{\nabla}$. Then, we have

(i)

$$
\tau_{S(T M)}(p) \leq n(n-1) c-2(n-1) \lambda+\varphi n^{2} \mu^{2}+\left(\frac{1+\varphi^{2}}{2}\right) \sum_{i, j=1}^{n}\left(m_{i j}\right)^{2} .
$$

(ii)

$$
\tau_{S(T M)}(p) \geq n(n-1) c-2(n-1) \lambda+\varphi n^{2} \mu^{2}-\left(\frac{1+\varphi^{2}}{2}\right) \sum_{i, j=1}^{n}\left(m_{i j}\right)^{2} .
$$

Theorem 4.6. Let $M$ be an $(n+1)$-dimensional lightlike hypersurface of a Lorentzian space form $\widetilde{M}(c)$ of constant sectional curvature $c$, endowed with a semi-symmetric metric connection $\widetilde{\nabla}$. Then, we derive

$$
\begin{aligned}
\tau_{S(T M)}(p) \leq & n(n-1) c-2(n-1) \lambda+\frac{1}{2}(\operatorname{trace} \bar{A})^{2}-\frac{1}{2}\left(\text { trace }_{N}\right)^{2} \\
& -\frac{1}{4} \sum_{i, j=1}^{n}\left(m_{i j}+D_{j i}\right)^{2}+\frac{1}{4} \sum_{i, j=1}^{n}\left(m_{i j}-D_{j i}\right)^{2}
\end{aligned}
$$


where

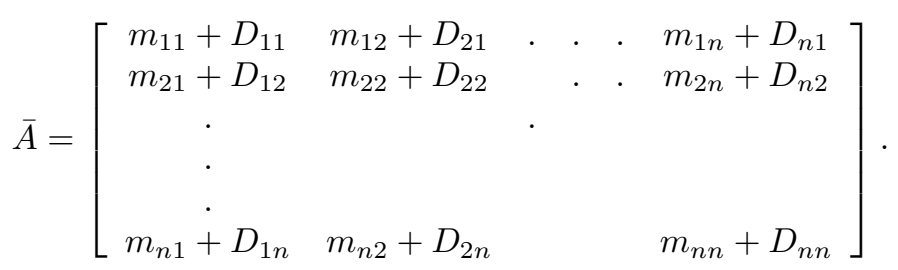

For all $p \in M$ the equality case of (4.42) satisfies iff $M$ is minimal.

Proof. From (4.7), (4.33) and (4.34) we obtain

$$
\begin{aligned}
\tau_{S(T M)}(p)= & n(n-1) c-2(n-1) \lambda+\frac{1}{2} \sum_{i, j=1}^{n}\left(m_{i i}+D_{j j}\right)^{2}-\frac{1}{2}\left(\sum_{i=1}^{n} m_{i i}\right)^{2} \\
& -\frac{1}{2}\left(\sum_{j=1}^{n} D_{j j}\right)^{2}-\frac{1}{2} \sum_{i, j=1}^{n}\left(m_{i j}+D_{j i}\right)^{2}+\frac{1}{2} \sum_{i, j=1}^{n}\left(m_{i j}\right)^{2}+\left(D_{j i}\right)^{2} .
\end{aligned}
$$

From (4.38) we have

$$
-\frac{1}{2} \sum_{i, j=1}^{n}\left(m_{i j}+D_{j i}\right)^{2}+\frac{1}{2} \sum_{i, j=1}^{n}\left(m_{i j}\right)^{2}+\left(D_{j i}\right)^{2}=-\frac{1}{4} \sum_{i, j=1}^{n}\left(m_{i j}+D_{j i}\right)^{2}+\frac{1}{4} \sum_{i, j=1}^{n}\left(m_{i j}-D_{j i}\right)^{2} .
$$

Using (4.45) in (4.44), we get

$$
\begin{aligned}
\tau_{S(T M)}(p)= & n(n-1) c-2(n-1) \lambda+\frac{1}{2} \sum_{i, j=1}^{n}\left(m_{i i}+D_{j j}\right)^{2}-\frac{1}{2}\left(\sum_{i=1}^{n} m_{i i}\right)^{2} \\
& -\frac{1}{2}\left(\sum_{j=1}^{n} D_{j j}\right)^{2}-\frac{1}{4} \sum_{i, j=1}^{n}\left(m_{i j}+D_{j i}\right)^{2}+\frac{1}{4} \sum_{i, j=1}^{n}\left(m_{i j}-D_{j i}\right)^{2} .
\end{aligned}
$$

Assume the equality case of (4.42) is satisfied, then

$$
\sum_{i} m_{i i}=0
$$

Thus $M$ is minimal.

Thus we get the following corollary.

Corollary 4.2. Let $M$ be an $(n+1)$-dimensional lightlike hypersurface of a Lorentzian space form $\widetilde{M}(c)$ of constant sectional curvature $c$, endowed with a semi-symmetric metric connection $\widetilde{\nabla}$. Then, we get

$$
\tau_{S(T M)}(p) \leq n(n-1) c-2(n-1) \lambda+\left(\frac{2 \varphi+1}{2}\right) n^{2} \mu^{2}-\varphi \sum_{i=1}^{n}\left(m_{i j}\right)^{2} .
$$

For all $p \in M$ the equality case of (4.47) satisfies iff $M$ is minimal.

Theorem 4.7. Let $M$ be an $(n+1)$-dimensional screen homothetic lightlike hypersurface of a Lorentzian space form $\widetilde{M}(c)$ of constant sectional curvature $c$, endowed with a semi-symmetric metric connection $\widetilde{\nabla}$. Then, we derive

$$
\begin{aligned}
\tau_{S(T M)}(p) \leq & n(n-1) c-2(n-1) \lambda+\frac{(n-1)}{2 n}(\operatorname{trace} \bar{A})^{2}-\frac{1}{2}\left(\text { trace }_{N}\right)^{2} \\
& -\frac{1}{2} n^{2} \mu^{2}-\frac{1}{2} \sum_{i \neq j}\left(m_{i j}+D_{j i}\right)^{2}+\frac{1}{2} \sum_{i, j=1}^{n}\left(m_{i j}\right)^{2}+\left(D_{j i}\right)^{2},
\end{aligned}
$$

where $\bar{A}$ is equal to (4.43).

For all $p \in M$ the equality case of (4.48) satisfies iff $n \mu=-$ trace $_{N}$. 
Proof. From (4.44),we get

$$
\begin{aligned}
\tau_{S(T M)}(p)= & n(n-1) c-2(n-1) \lambda+\frac{1}{2}(\operatorname{trace} \bar{A})^{2}-\frac{1}{2}\left(\text { trace }_{N}\right)^{2}-\frac{1}{2} n^{2} \mu^{2} \\
& -\frac{1}{2} \sum_{i=1}^{n}\left(m_{i i}+D_{i i}\right)^{2}-\frac{1}{2} \sum_{i \neq j}\left(m_{i j}+D_{j i}\right)^{2}+\frac{1}{2} \sum_{i, j=1}^{n}\left(m_{i j}\right)^{2}+\left(D_{j i}\right)^{2} .
\end{aligned}
$$

Using Lemma 4.1 and equality case of (4.49), we have

$$
\begin{aligned}
\tau_{S(T M)}(p) \leq & n(n-1) c-2(n-1) \lambda+\frac{1}{2}(\operatorname{trace} \bar{A})^{2}-\frac{1}{2}\left(\operatorname{trace}_{N}\right)^{2}-\frac{1}{2} n^{2} \mu^{2} \\
& -\frac{1}{2 n}\left(\sum_{i=1}^{n} m_{i i}+D_{i i}\right)^{2}-\frac{1}{2} \sum_{i \neq j}\left(m_{i j}+D_{j i}\right)^{2}+\frac{1}{2} \sum_{i, j=1}^{n}\left(m_{i j}\right)^{2}+\left(D_{j i}\right)^{2}
\end{aligned}
$$

which implies (4.48). The equality case of (4.48) holds, then

$$
m_{11}+D_{11}=m_{22}+D_{22}=\ldots=m_{n n}+D_{n n} .
$$

From (4.51) we obtain

$$
\begin{array}{cc}
(1-n) m_{11}+m_{22}+\ldots+m_{n n}+(1-n) D_{11}+D_{22}+\ldots+D_{n n}= & 0 \\
m_{11}+(1-n) m_{22}+\ldots+m_{n n}+D_{11}+(1-n) D_{22}+\ldots+D_{n n}= & 0 \\
& \cdot \\
& \cdot \\
m_{11}+m_{22}+\ldots+(1-n) m_{n n}+D_{11}+D_{22}+\ldots+(1-n) D_{n n}= & 0 .
\end{array}
$$

Using last equations, we have

$$
(n-1)^{2}\left(\operatorname{trace} A_{N}+n \mu\right)=0 .
$$

Because of $n \neq 1$,we get $n \mu=-\operatorname{trace} A_{N}$.

Thus we get the following corollary.

Corollary 4.3. Let $M$ be an $(n+1)$-dimensional screen homothetic lightlike hypersurface of a Lorentzian space form $\widetilde{M}(c)$ of constant sectional curvature $c$, endowed with a semi-symmetric metric connection $\widetilde{\nabla}$. Then, we have

$$
\tau_{S(T M)}(p) \leq n(n-1) c-2(n-1) \lambda+\varphi n(n-1) \mu^{2}-\frac{\left(1+\varphi^{2}\right)}{2} n \mu^{2}-\varphi \sum_{i \neq j}^{n}\left(m_{i j}\right)^{2}+\frac{\left(1+\varphi^{2}\right)}{2} \sum_{i=1}^{n}\left(m_{i i}\right)^{2} .
$$

For all $p \in M$ the equality case of (4.53) satisfies iff either $\varphi=-1$ or $M$ is minimal.

Theorem 4.8. Let $M$ be an $(n+1)$-dimensional lightlike hypersurface of a Lorentzian space form $\widetilde{M}(c)$ of constant sectional curvature $c$, endowed with a semi-symmetric metric connection $\widetilde{\nabla}$. Then

$$
\begin{aligned}
\tau_{S(T M)}(p) \geq & n(n-1) c-2(n-1) \lambda+\frac{1}{2}\left((\operatorname{trace} \bar{A})^{2}-\left(\operatorname{trace}_{N}\right)^{2}-n(n-1) \mu^{2}\right) \\
& +\frac{1}{2}\left(\sum_{i \neq j=1}^{n}\left(m_{i j}\right)^{2}-\sum_{i, j=1}^{n}\left(m_{i j}+D_{j i}\right)^{2}+\sum_{i, j=1}^{n}\left(D_{j i}\right)^{2}\right) .
\end{aligned}
$$

For all $p \in M$ the equality case of (4.54) satisfies iff $p$ is a totally umbilical point.

Proof. Using (4.44),we get

$$
\begin{aligned}
\tau_{S(T M)}(p)= & n(n-1) c-2(n-1) \lambda+\frac{1}{2}(\operatorname{trace} \bar{A})^{2}-\frac{1}{2}\left(\text { trace } A_{N}\right)^{2}-\frac{1}{2} n^{2} \mu^{2} \\
& +\frac{1}{2} \sum_{i=1}^{n}\left(m_{i i}\right)^{2}+\frac{1}{2} \sum_{i \neq j=1}^{n}\left(m_{i j}\right)^{2}+\frac{1}{2} \sum_{i, j=1}^{n}\left(D_{j i}\right)^{2}-\frac{1}{2} \sum_{i, j=1}^{n}\left(m_{i j}+D_{j i}\right)^{2} .
\end{aligned}
$$


Using Lemma 4.2 and equality case of (4.44), we have

$$
\begin{aligned}
\tau_{S(T M)}(p) \geq & n(n-1) c-2(n-1) \lambda+\frac{1}{2}(\operatorname{trace} \bar{A})^{2}-\frac{1}{2}\left(\operatorname{trace} A_{N}\right)^{2}-\frac{1}{2} n^{2} \mu^{2} \\
& -\frac{1}{2} \sum_{i, j=1}^{n}\left(m_{i j}+D_{j i}\right)^{2}+\frac{1}{2 n}\left(\sum_{i=1}^{n} m_{i i}\right)^{2}+\frac{1}{2} \sum_{i \neq j=1}^{n}\left(m_{i j}\right)^{2}+\frac{1}{2} \sum_{i, j=1}^{n}\left(D_{j i}\right)^{2}
\end{aligned}
$$

which implies (4.53). The equality case of (4.43) satisfies iff

$$
m_{11}=\ldots=m_{n n}
$$

and the shape operator $A_{\xi}^{*}$ becomes of the form

$$
A_{\xi}^{*}=\left[\begin{array}{ccccccc}
m_{11} & 0 & . & . & . & 0 & 0 \\
0 & m_{11} & \cdot & \cdot & . & 0 & 0 \\
\cdot & & \cdot & & & & \\
\cdot & & & . & & & \\
\cdot & & & & . & & \\
0 & 0 & . & . & . & m_{11} & 0 \\
0 & 0 & . & . & . & 0 & 0
\end{array}\right] \text {, }
$$

which indicates that $M$ is totally umbilical. Hence, the claim holds.

Thus we get the following corollary.

Corollary 4.4. Let $M$ be an $(n+1)$-dimensional screen homothetic lightlike hypersurface of a Lorentzian space form $\widetilde{M}(c)$ of constant sectional curvature $c$, endowed with a semi-symmetric metric connection $\widetilde{\nabla}$. Then, we get

$$
\tau_{S(T M)}(p) \geq n(n-1) c-2(n-1) \lambda+\frac{(2 \varphi+1)}{2} n^{2} \mu^{2}-\frac{n(n-1)}{2} \mu^{2}-\frac{(2 \varphi+1)}{2} \sum_{i, j=1}^{n}\left(m_{i j}\right)^{2} .
$$

For all $p \in M$ the equality case of (4.58) satisfies iff $p$ is a totally umbilical point.

\section{Acknowledgments}

The authors have greatly benefited from the referee's report. So we wish to express our gratitude to the reviewer for his/her valuable suggestions which improved the content and presentation of the paper.

\section{References}

[1] Atindogbe, C. and Duggal, Krishan L., Conformal screen on lightlike hypersurfaces. Int. J. Pure Appl. Math., 11(2004),4,421-442.

[2] Beem, J. K., Ehrlich, P. E., Easley, K. L., Global Lorentzian Geometry. Dekker, New York, 1996.

[3] Bejan, C. L. and Duggal, Krishan L., Global lightlike manifolds and harmonicity. Kodai Math. J., 28(2005), 1, 131-145.

[4] Chen, B. Y., Mean curvature and shape operator of isometric immersion in real space forms. Glasgow Mathematic Journal, 38 (1996), 87-97.

[5] Chen, B. Y., Relation between Ricci curvature and shape operator for submanifolds with arbitrary codimension. Glasgow Mathematic Journal, 41(1999), 33-41.

[6] Chen, B. Y., Some pinching and classification theorems for minimal submanifolds. Arch. math. (Basel), 60(1993), 6, 568-578.

[7] Chen, B. Y., A Riemannian invariant and its applications to submanifold theory. Result Math., 27(1995), 17-26.

[8] Chen, B. Y., Dillen, F., Verstraelen L. and Vrancken, V., Characterizations of Riemannian space forms, Einstein spaces and conformally flat spaces. Proc. Amer. Math. Soc., 128(2000),589-598.

[9] Chen, B. Y., A Riemannian invariant for submanifolds in space forms and its applications. Geometry and Topology of submanifolds $V I$, (Leuven, 1993/Brussels, 193), (NJ:Word Scientific Publishing, River Edge), 1994, pp.58 - 81, no.6, 568 - 578.

[10] Duggal, Krishan L., On scalar curvature in lightlike geometry. Journal of Geometry and Physics, 57(2007), 2, 473-481.

[11] Duggal, Krishan L. And Bejancu, A., Lightlike Submanifold of Semi-Riemannian Manifolds and Applications. Kluwer Academic Pub., The Netherlands, 1996.

[12] Duggal, Krishan L. and Şahin, B., Differential Geometry of Lightlike Submanifolds. Birkhauser Verlag AG., 2010.

[13] Duggal, Krishan L. and Sharma, R., Semi-Symmetric metric connection in a Semi-Riemannian Manifold. Indian J. Pure appl Math., 17(1986), 1276-1283. 
[14] Gülbahar, M., Kılıç, E. and Keleş, S., Chen-like inequalities on lightlike hypersurfaces of a Lorentzian manifold. J. Inequal. Appl., 2013:266,18pp.

[15] Gülbahar, M., Kılıç, E. and Keleş, S., Some inequalities on screen homothetic lightlike hypersurfaces of a lorentzian manifold. Taizwanese Journal of Mathematics, 17(2013), 2, 2083-2100.

[16] Güneş, R., Şahin, B. and Kılıç, E., On Ligtlike Hypersurfaces of a Semi-Riemannian Space Form. Turk J. Math., 27(2003), $283-297$.

[17] Hayden, H. A., Subspace of a space with Torsion. Proc. London Math. Soc., 34(1932), 27-50.

[18] Hong, S., Matsumoto, K. and Tripathi, M. M., Certain basic inequalities for submanifolds of locally conformal Kaehlerian space forms. SUT J. Math., 4(2005), 1, 75-94.

[19] Imai, T., Notes on Semi-Symmetric Metric Connection. Tensor, N.S., 24(1972), 293-296.

[20] Imai, T., Hypersurfaces of a Riemannian Manifold with Semi-Symmetric Metric Connection. Tensor, N.S., 23(1972), 300-306.

[21] Liu, X. and Zhou, J., On Ricci curvature of certain submanifolds in cosympletic space form. Sarajeva J. Math., 2(2006), 95-106.

[22] Konar, A. and Biswas, B., Lorentzian Manifold that Admits a type of Semi-Symmetric Metric Connection. Bull. Cal. Math., Soc., 93(2001), No.5, 427-437.

[23] Mihai, A. and Özgür, C., Chen inequalities for submanifolds of real space form with a semi-symmetric metric connection. Taiwanese Journal of Mathematics, 14(2010), No. 4, pp. 1465-1477.

[24] O'Neill, B., Semi-Riemannian Geometry with Applications to Relativity. Academic Press. London, 1983.

[25] (Önen) Poyraz, N. and Yaşar, E., Chen-like inequalities on lightlike hypersurface of a Lorentzian product manifold with a quartersymmetric nonmetric connection. Kragujevac Journal of Mathematics, 40 (2016), 2, 146-164.

[26] Tripathi, M. M., Improved Chen-Ricci inequality for curvature-like tensor and its applications. Differential Geom. Appl., 29(2011), 685-698.

[27] Yano, K., On Semi-Symmetric Metric Connection. Rev. Roum. Math.Pures Et Appl., 15 (1970), 1579-1586.

[28] Yaşar, E., Çöken, A. C. and Yücesan, A., Lightlike Hypersurfaces of Semi-Riemannian Manifold with Semi-Symmetric Metric Connection. Kuweyt Journal of Science and Engineering, 34 (2007), 11-24.

\section{Affiliations}

\section{NERGiz (ÖNEN) POYRAZ}

ADDREsS: Çukurova University, Dept. of Mathematics, 01330, Adana-Turkey.

E-MAIL: nonen@cu.edu.tr

\section{BURÇIN DOĞAN}

ADDRESS: Mersin University, Dept. of Mathematics, 33110, Mersin-Turkey.

E-MAIL: bdogan@mersin.edu.tr

\section{EROL YAŞAR}

AdDRESS: Mersin University, Dept. of Mathematics, 33110, Mersin-Turkey.

E-MAIL: yerol@mersin.edu.tr 